\title{
Resilient Design of Recharging Station Networks for Electric Transportation Vehicles
}

\section{4th International Symposium on Resilient Control Systems}

Kris Villez

Akshya Gupta

Venkat Venkatasubramanian

Craig Rieger

August 2011

The INL is a

U.S. Department of Energy

National Laboratory

operated by

Battelle Energy Alliance

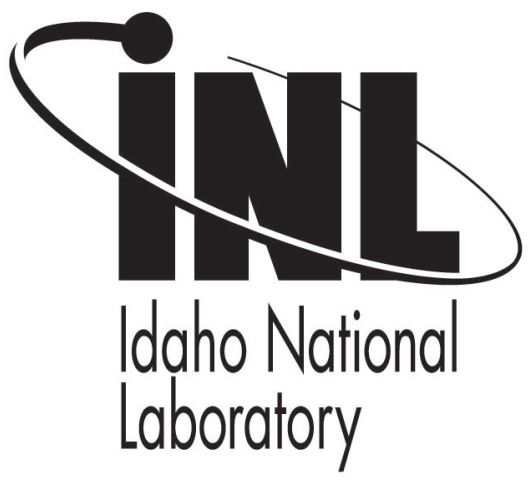

This is a preprint of a paper intended for publication in a journal or proceedings. Since changes may be made before publication, this preprint should not be cited or reproduced without permission of the author. This document was prepared as an account of work sponsored by an agency of the United States Government. Neither the United States Government nor any agency thereof, or any of their employees, makes any warranty, expressed or implied, or assumes any legal liability or responsibility for any third party's use, or the results of such use, of any information, apparatus, product or process disclosed in this report, or represents that its use by such third party would not infringe privately owned rights. The views expressed in this paper are not necessarily those of the United States Government or the sponsoring agency. 


\section{Resilient design of recharging station networks for electric transportation vehicles}

\author{
Kris Villez, Akshya Gupta, Venkat \\ Venkatasubramanian \\ School of Chemical Engineering \\ Purdue University \\ West Lafayette, IN, USA \\ $\{$ kvillez,gupta72,venkat\}@purdue.edu
}

\author{
Craig Rieger \\ Idaho National Laboratory \\ PO Box 1625, \\ Idaho Falls, ID, USA \\ craig.rieger@inl.gov
}

\begin{abstract}
As societies shift to "greener" means of transportation using electricity-driven vehicles one critical challenge we face is the creation of a robust and resilient infrastructure of recharging stations. A particular issue here is the optimal location of service stations. In this work, we consider the placement of battery replacing service station in a city network for which the normal traffic flow is known. For such known traffic flow, the service stations are placed such that the expected performance is maximized without changing the traffic flow. This is done for different scenarios in which roads, road junctions and service stations can fail with a given probability. To account for such failure probabilities, the previously developed facility interception model is extended. Results show that service station failures have a minimal impact on the performance following robust placement while road and road junction failures have larger impacts which are not mitigated easily by robust placement.
\end{abstract}

Robustness, Resilience, Network optimization, electrical transportation, Facility location

\section{INTRODUCTION}

A systematic and generic approach to the design and operation of networked systems has gained attention in recent publications [1-6], following important results in the analysis of networks [7-8]. In some of our recent publications, it has been shown that efficiency and robustness are important considerations in the design of networks, leading to different optimal topologies [9-10]. In this work, we take this into account to search the optimal location of service stations for electrical vehicles.

Electrical vehicles constitute a technology that has made significant progress in the last decade to the point where gradual replacement of the vehicle fleet becomes realistic. Important advantages of electrical vehicles include (1) more efficient use of energy [11], thereby reducing the need for energy resources, (2) flexibility in the choice for the source of energy to be used for electricity generation, thereby reducing the need for environmentally unfriendly resources and (3) the reduction of diffuse pollution as produced by conventional vehicles which is at least partly replaced by concentrated pointwise pollution which is easier to manage (e.g. coal, gas or nuclear based electricity plants). Despite these advantages, the successful transition to a society with a prominent role for electrical transportation still requires considerable changes in available infrastructure and corresponding investments. For example, an electrical vehicle needs frequent replacement or recharging of its battery for which service stations are necessary. In addition, the travel range of an electrical vehicle is rather small compared to conventional cars [12], making the desired amount of such service location considerable large. We consider the replacement of a used battery with a charged one [13] the approach of choice as this does not require a long-term stationary period. Electrical vehicles need to visit service stations to replace a used battery in an electrical car with a recharged one. To reduce the loss of energy due to such visits, the stations are to be located in places which require minimal adjustments in the normal traffic flow as expected for conventional cars.

The above mentioned problem can be described by a modified version of the facility-interception model [14] and solved by means of an Integer Linear Programming (ILP) approach [15]. In this work, we now consider that parts of the infrastructure (road, road junctions and service stations) may fail. To this end, we apply a particular failure rate for roads, junctions and service stations and optimize the resulting expected performance of the whole infrastructure by optimization the location of service stations. This requires further adjustment of the facility-interception model which makes the problem computationally intensive in its complete formulation. To reduce computational time, we consider only the most important fraction of the paths taken through the network. Our method is tested on the city of Alexandria, VA. as a case study.

\section{Materials and Methods}

\section{A. Case study}

The optimization of the service station locations requires that one knows the expected traffic flow in a given network. One strategy to obtain such flows is to execute an extensive monitoring campaign by which one measures the flows on major roads and estimates flows in unmeasured sections of the network, either by interpolation or extrapolation. Needless to say that this is expensive and time-consuming. A second 
strategy mitigates such problems by means of agent-based modeling. Based on general expectations and distribution of the residing population over the road network, agent-based modeling allows to generate expected traffic flows. In this study, we use the TRANSIMS software developed by Los Alamos National Laboratory to forecast expected traffic flows. This was previously done for Portland, Oregon [16] and Chittenden County, Vermont [17]. We use the city of Alexandria, VA, as a case study to test our optimization method and software which is also available in TRANSIMS and as used in prior work [15]. Figure 1 shows the road network for this case study. Following the agent-based model simulation, we obtain (1) the topology of the traffic network including arcs (roads) and nodes (road junctions or terminal ends), (2) the routes (paths) in the network with non-zero traffic flow, (3) the traffic flow in each identified route. The road network has 2620 nodes and 3653 arcs. The number of unique paths is 114941 and the total flow 384584 cars per day.

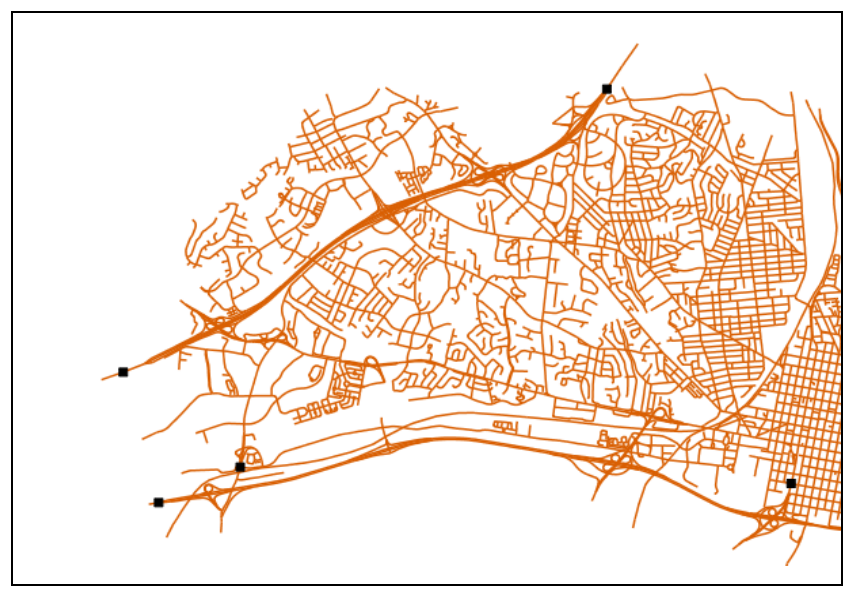

Figure 1. Road map of Alexandria, VA, with 5 service stations (squares)

TABLE I. EVALUATED SCENARIOS FOR ROBUST FACILITY PLACEMENT

\begin{tabular}{|l|l|l|l|l|}
\hline \multirow{2}{*}{ Scenario } & \multirow{2}{*}{ Description } & \multicolumn{4}{|c|}{ Failure rate [\%] } \\
\cline { 3 - 5 } & & Arc & Node & Service station \\
\hline 1 & No failure & 0 & 0 & 0 \\
\hline 2 & Arc failures & 1 & 0 & 0 \\
\hline 3 & Arc failures & 5 & 0 & 0 \\
\hline 4 & Node failures & 0 & 1 & 0 \\
\hline 5 & Node failures & 0 & 5 & 0 \\
\hline 6 & Service station failures & 0 & 0 & 1 \\
\hline 7 & Service station failures & 0 & 0 & 5 \\
\hline
\end{tabular}

\section{B. Infrastructural failures}

We optimize the location of service stations for several scenarios as listed in Table 1. First, we consider a scenario where all infrastructure elements (arcs, nodes and service stations) show no failures, i.e. the failure rates for nodes $\left(\rho_{\mathrm{a}}\right)$, $\operatorname{arcs}\left(\rho_{\mathrm{n}}\right)$ and service stations $\left(\rho_{\mathrm{s}}\right)$ are all set to $0 \%$. In our current simulations, these are considered fixed across the network although they can be set individually in the RNEDE software implementation [6]. We optimize the location of 1 up to 20 stations under these conditions. The obtained results are used as a reference. Six additional scenarios are also considered in which elements of one class only (arc, node, service station) can fail. I.e., we consider scenarios where either arcs, nodes or service stations are expected to fail but never together. By doing so, we investigate the impact of the failures of individual element classes on the design and expected performance. The applied failure rates are $1 \%$ and $5 \%$ respectively.

\section{Robust facility placement}

A transportation network can be abstracted to a graph representation, denoted $\mathrm{G}(\mathrm{N}, \mathrm{A})$. In such representation, the set of nodes, $\mathrm{N}$, consists of road end points (terminal nodes) as well as road junctions (non-terminal nodes) whereas the arcs, A, represent the roads between nodes. A path is then defined as a specified route from a given travel origin to a travel destination. We define the set $\mathrm{P}$ as the set of paths, $\mathrm{p}$, with a non-zero traffic flow. For each of the paths in this subset, the traffic flow is noted as $f_{p}\left(f_{p}>0\right)$. The total flow in the given network $\left(\mathrm{f}_{\text {total }}\right)$ is then written as:

$$
f_{\text {total }}=\sum_{p \in P} f_{p}
$$

Now, consider that one needs to locate a given number of recharging facilities on the network so that the maximum fraction of this flow in the network is intercepted without need for re-routing. We write:

$$
\begin{array}{rll}
x_{j} & =1 & \text { if a service station is located on node } j \\
& =0 & \text { otherwise }
\end{array}
$$

Then the overall objective function to be maximized is the fraction of the total flow that is expected to be intercepted without changing the flow pattern. This objective function is written as follows:

$$
J=\frac{1}{f_{\text {total }}} \sum_{p \in P} f_{p} \cdot y_{p} \cdot \rho_{p}
$$

with $\mathrm{y}_{\mathrm{p}}$ representing the presence of a service station on the path p:

$$
\begin{array}{rll}
y_{p} & =1 \quad \text { if a service station is located on path } p \\
& =0 & \text { otherwise }
\end{array}
$$

and $\rho_{\mathrm{p}}$ equal to the probability that all elements in the path are functioning well. Quite naturally, it follows that:

$$
\sum_{j \in p} x_{j} \geqslant y_{p}
$$

We write the probability $\rho_{\mathrm{p}}$ as follows: 


$$
\rho_{p}=1-\left(\rho_{a} \cdot m_{a, p}+\rho_{n} \cdot m_{n, p}+\rho_{s} \cdot m_{s, p}\right)
$$

with $\rho_{\mathrm{a}}, \rho_{\mathrm{n}}$ and $\rho_{\mathrm{s}}$ the failure probabilities as described before. The variables $\mathrm{m}_{\mathrm{a}, \mathrm{p}}, \mathrm{m}_{\mathrm{n}, \mathrm{p}}$ and $\mathrm{m}_{\mathrm{s}, \mathrm{p}}$ are the integer numbers of respectively nodes, arcs and refueling stations on the considered path $\mathrm{p}$. The integers $\mathrm{m}_{\mathrm{a}, \mathrm{p}}$ and $\mathrm{m}_{\mathrm{n}, \mathrm{p}}$ are fixed for a given road network. In contrast, $\mathrm{m}_{\mathrm{s}, \mathrm{p}}$, follows from a proposed placement of refueling stations. This variable is not binary and is not identified upfront, which means the model does not represent an Integer Linear program (ILP) anymore. To reformulate the problem as an ILP, we rewrite equation 6 as follows:

$$
\rho_{p}=1-\left(\rho_{n} \cdot m_{n, p}+\rho_{a} \cdot m_{a, p}+\sum_{m=1}^{m_{n, p}} z_{s, p, m} \cdot \rho_{s}^{m}\right)
$$

with

$$
\begin{array}{rll}
z_{s, p, m}=1 & \text { if } m \text { service stations are } \\
& \text { located on path } p \\
=0 & \text { otherwise }
\end{array}
$$

These newly introduced binary variables $\mathrm{z}_{\mathrm{s}, \mathrm{p}, \mathrm{m}}$ are subject to following constraints:

$$
\sum_{m=1}^{m_{n, p}} z_{s, p, m} \leqslant 1
$$

The facility location problem is now solved by maximization of $\mathbf{J}$ (equation 3 ) by manipulation of the binary variables, $x_{j}, y_{p}, z_{s, p, m}$ subject to constraint equations (5) and (9). Consider now that the total number of facilities to be placed is equal to $\mathrm{m}_{\mathrm{s}, \text { total }}$. Then the solution is further constrained as follows:

$$
\sum_{j=1}^{n} x_{j} \leqslant m_{s, t o t a l}
$$

The above model is an extension of the model used in [15] to include failure probabilities for individual elements in the network. The model evaluates the expected performance under such probabilities. The model of [15] was in turn based on the classic flow interception facility location model by [14]. The model is implemented in General Algebraic Modeling System (GAMS, v23.2.1) coupled with the ILOG CPLEX solver (v12.1.0). For the location of 20 service stations, the problem contains 2298820 variables $\left(\mathrm{x}_{\mathrm{j}}(2620), \mathrm{y}_{\mathrm{p}}(114941)\right.$ and $\mathrm{z}_{\mathrm{s}, \mathrm{p}, \mathrm{m}}$ (114941 paths x maximally 20 stations) ) and 22983 constraints (equation 5 and 8 for each path $\mathrm{p}$ and one for equation 9). This problem is computationally very intensive due to the increased number of decision variables. In particular, the binary decision variables $z_{\mathrm{s}, \mathrm{p}, \mathrm{m}}$ are new compared to the original problem as formulated in [15]. As a result, the time needed to solve this problem is considerably large. To scale this down, we solve a reduced form of the above problem within this work as follows.
First, we define a subset of paths $P_{R}$ as the set of paths with a traffic flow higher or equal to a critical integer number, $f_{\text {crit }}$ :

$$
p \in P_{R} \Leftrightarrow f_{p} \geq f_{c r i t}
$$

and the flow over this subset $\left(\mathrm{f}_{\mathrm{R}}\right)$ :

$$
f_{R}=\sum_{p \in P_{R}} f_{p}
$$

Now, a reduced objective function is defined over this subset alone:

$$
J_{R}=\frac{1}{f_{R}} \sum_{p \in P_{R}} f_{p} \cdot y_{p} \cdot \rho_{p}
$$

Now, we optimize $J_{R}$ instead of the original objective function $J$ with the same solver. In our study, we use a critical flow ( $\left.f_{\text {crit }}\right)$ of 5 cars per day. The number of paths in the subset $P_{R}$ is 9865 (8.6\% of original). The traffic flow over this subset $\left(f_{R}\right)$ amounts to 239278 cars per day, which is $62 \%$ of the original, total flow. As such, it is clear that a fair reduction of the size of mathematical programming problem still permits to take the majority of the traffic flow into account. The total number of variables is now 209785 and the number of constraints 19731.

\section{Results}

For all of the evaluated scenarios and for all number of service stations, the service station locations were optimized. As an example, Figure 1 shows the resulting service station locations for scenario 1 (no failures) with 5 service stations. In what follows, the optimized expected performance is analyzed first as function of number of stations and failure rates. Next, maps are shown for different scenarios and observed patterns are explained.

\section{A. Optimized performance}

Figure 2 shows the expected performance, i.e. the expected fraction of intercepted cars without changing the normal traffic flow, as obtained following optimization with the above method. As can be expected, the highest line corresponds to the scenario with no failures at all. One can see that the performance increases from about $40 \%$ for 1 station to over $95 \%$ with 20 stations which is not very surprising either. In addition, one can see that the marginal increase in performance decreases with each added service station. The performances obtained for failures of the service stations are very close to those obtained for the failure-free scenario and show a very similar trend. This means (1) that service station failure affects the expected performance only marginally, (2) that the design method can mitigate the effects of station failures well by means of the optimization method or (3) both. We investigate this further below. The lowest performance values are found for the scenarios with 5\% arc failure and with $5 \%$ node failure. These failure rates have a dramatic impact as the performance now ranges from about $12 \%$ for 1 service station to about $33 \%$ 
for 20 service stations. This shows that the failures in the road network are more important than failures of service stations if one assumes similar failure rates for either one of them. It also shows that robust optimization of the location of service stations is likely not enough to mitigate road network failures. In between the results for the normal scenario and the scenarios with $5 \%$ failure rates for either arcs or nodes, one can observe the results for the scenarios for $1 \%$ failure rates in either arcs or nodes of the road network, as expected. The obtained values (from about $30 \%$ to about $74 \%$ ) suggest that the effect of the failure rates is concave. I.e., a change of the failure rate at lower values for the failure rate has a higher marginal effect than at higher values.

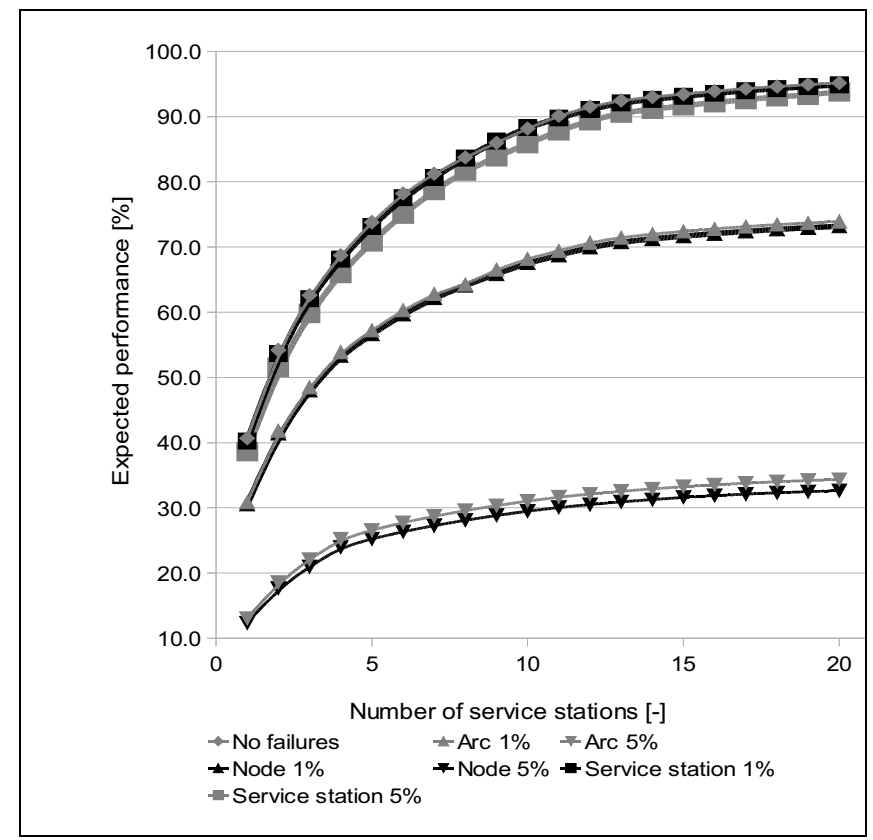

Figure 2. Optimized expected performance as function of the number of installed stations for different failure scenarios.

\section{B. Detailed study of resulting locations}

Figure 3 and 4 show the maps for scenario 1 for 15, resp. 20 service stations placed in the road network. One can compare these as well with Figure 1, where 5 service stations are placed. It can be seen that certain stations are kept in the same location which suggests that a hierarchical manner of placing additional service stations may be possible, instead of the brute-force Integer Programming approach. In addition, most station locations are found in a close neighborhood of the highways, which are shown as thick lines, one in the North and one in the South part of the city. This further suggests that a reduced approach, in which the number of candidate locations is reduced may be feasible.

The maps for all other scenarios were investigated in detail for the cases where 5,15 and 20 service stations were placed to investigate any observable effects of the failure rates on service station locations.. In the case of arc failures, some of service station locations change for each applied failure rate $(1 \%, 5 \%)$. Figure 5 shows the locations for 15 stations with $1 \%$ failure rate. Compared to the no failure scenario (scenario 1, Figure 3), four stations are found at a different location. In particular, one station originally on the highway at the south is now placed on a road accessing this highway. Two stations in the North are relocated to the West region of the city. In this region, six service stations are now found in stead of four for the no failure case. Of these six, three are at their original location. In the South-East of the city, the service station locations remain the same.

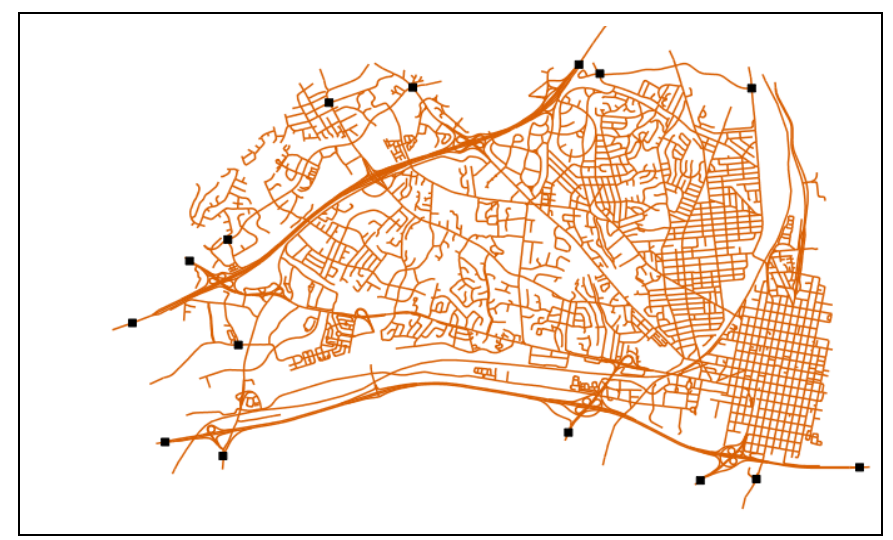

Figure 3. Optimized location of 15 service stations for scenario 1

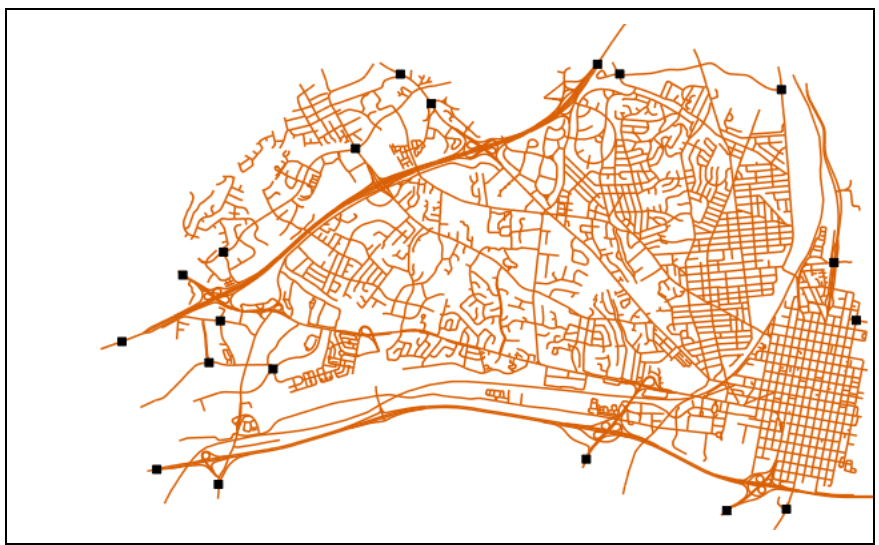

Figure 4. Optimized location of 20 service stations for scenario 1

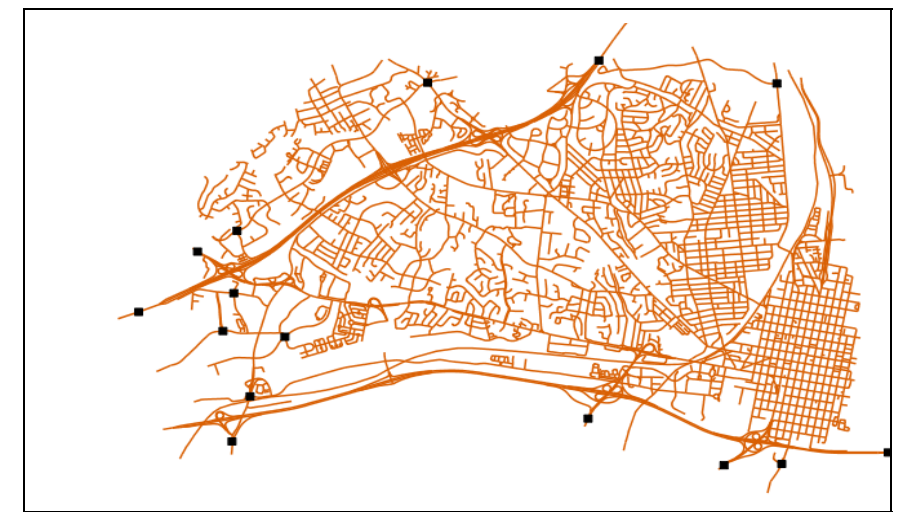

Figure 5. Optimized location of 15 service stations for scenario 2 (arc failure rate $1 \%$ ) 


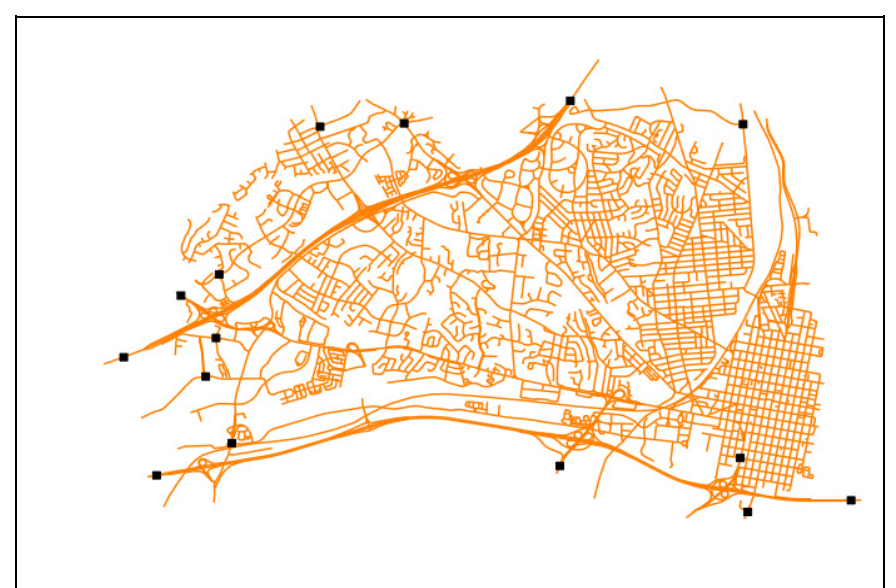

Figure 6. Optimized location of 15 service stations for scenario 3 (arc failure rate $5 \%$ )

At arc failures of $5 \%$ (scenario 3), the service station location change again (Figure 6). Compared to scenario 1 (Figure 3), ten stations remain at their location; compared to scenario 2 (Figure 5), twelve stations remain at their location. In scenario 3, one can now find four stations in the North. In the West, five remain at their location while one is removed. In the South, one can now find 2 stations on the highway again and one additional service station changes location, towards the inside of the city. Similar effects were observed for the arc failure scenarios with 5 and 20 stations placed. While they can be described in similar terms, it is however hard to draw a general line in these results.

Figure 7 shows the results for a $1 \%$ node failure rate (scenario 4). Compared to the results for scenario 1 (Figure 4), one can see that one station is taken from the West and one from the East and placed in the North-West region, where 5 stations are now placed fairly close to each other. Furthermore, the two stations located close to each other in the North-East region in Figure 4 are now further apart. For a 5\% node failure rate, results are shown in Figure 8. Now, the two stations added in the North for the $1 \%$ failure rate are now located in the West, though located towards the inner city. The results for 5 and 15 service stations are similar.

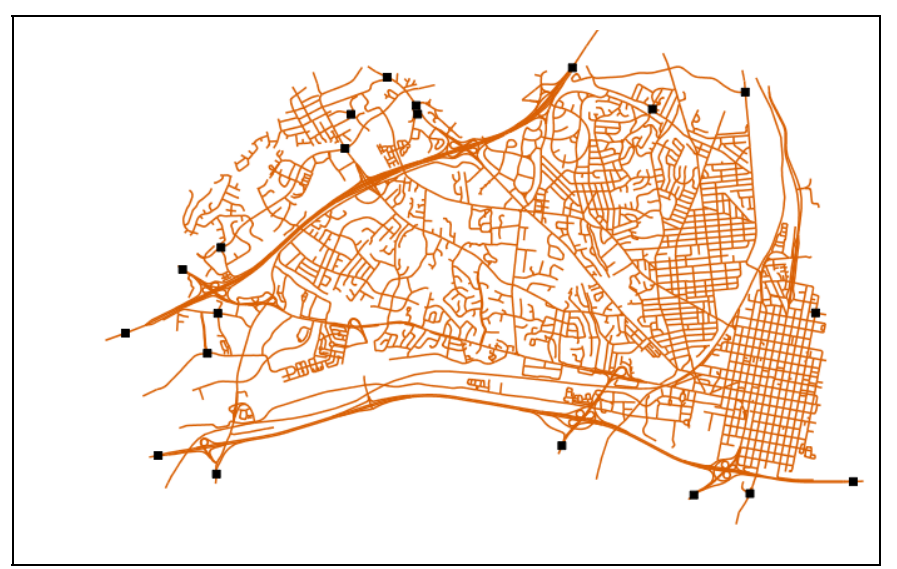

Figure 7. Optimized location of 20 service stations for scenario 4 (node failures rate $1 \%$ )

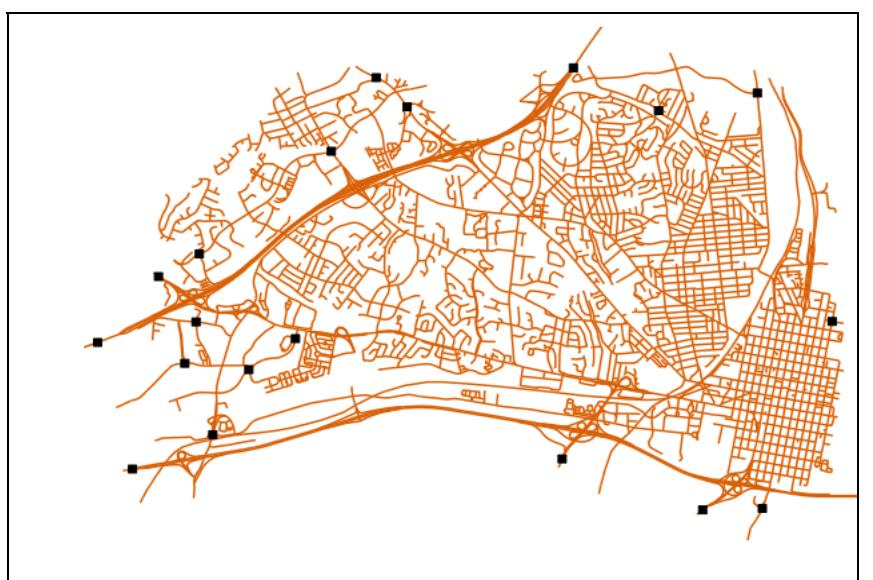

Figure 8. Optimized location of 20 service stations for scenario 5 (node failures rate 5\%)

Station failures were also investigated. For a $1 \%$ failure rate, the resulting locations for 15 and 20 stations are the same as obtained for the no failures scenario (Figure 3 and 4). For five stations, one service station is relocated as can be observed from Figure 9, when compared to Figure 1 . For a 5\% failure rate (scenario 7), the service station locations are the same again as in Figure 1. Figure 10 shows the locations for scenario 7 with 15 placed service stations. One can see that two stations are relocated. One is located the North-West region (Figure 3) is moved further towards the North. The other one is located in the North-West and is moved to a location on the highway in the North. In the case of 20 stations (Figure 11), three stations only are relocated compared to the no failure case (Figure 4). In the North-West, two stations are moved so that the group of three stations are found closer to each other. One station in the East (Figure 4) is moved to the West region though towards the inner part of the city. It is thus clear that accounting for failures in the service stations does lead to different optimal locations. As such, one can conclude that the limited effect on the expected performance of the failure rate is mitigated to some extend by facility relocation.

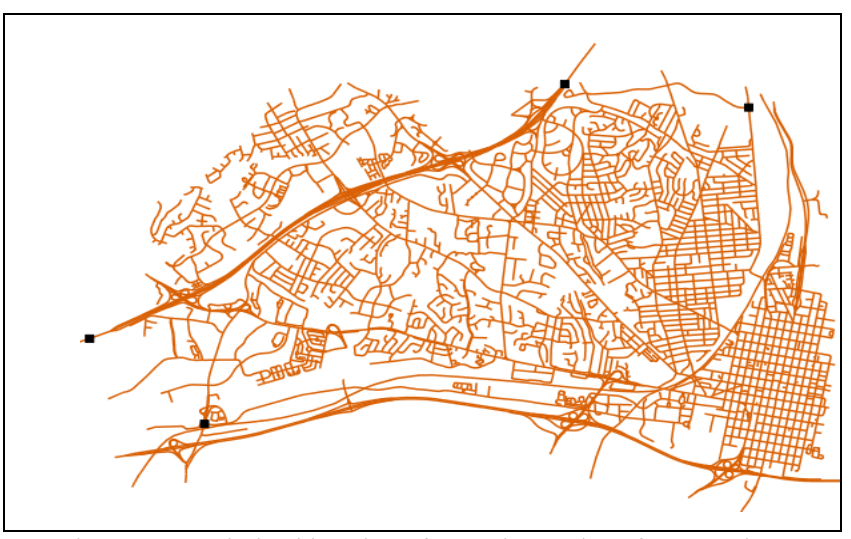

Figure 9. Optimized location of 5 service stations for scenario 6 (service station failure rate $1 \%$ ) 


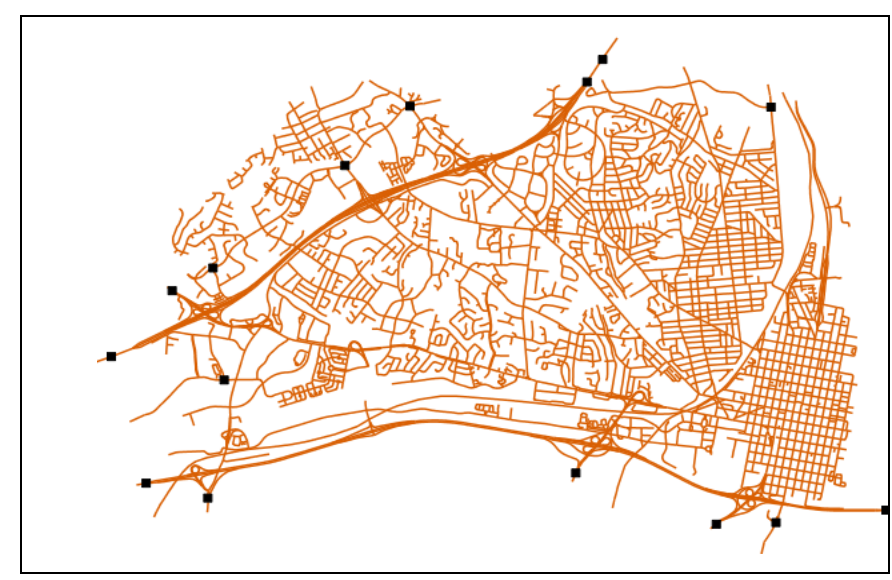

Figure 10. Optimized location of 15 service stations for scenario 7 (service station failure rate 5\%)

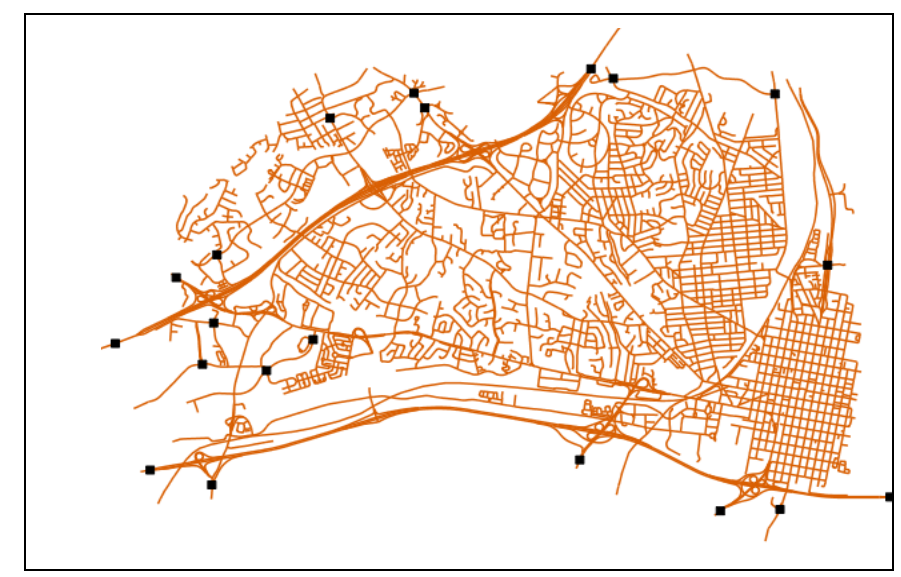

Figure 11. Optimized location of 20 service stations for scenario 7 (service station failure rate $5 \%$ )

\section{Conclusions}

In this work, a systematic approach to the location of service stations for recharging of electric vehicles is applied. The applied facility-interception model is extended to account for failures in infrastructural elements such as arcs (roads), nodes (road junctions) and facilities (service stations). Results indicate that failures of arcs and nodes have a large impact on the resulting performance which cannot be mitigated easily by facility location optimization without changing the flow pattern. The situation is different for failure rates of the service stations. In this case, a performance close to the original performance can be obtained by means of the proposed mathematical program. One of the most important assumptions is that the locations are to be optimized for the given flow pattern. This makes sense indeed for the scenario for no failures, which is expected to prevail most of the time. For the scenarios with failures, it is however easy to see that a temporary rerouting of the flow may be easier to achieve. This is currently under investigation and will be presented in future work.

\section{ACKNOWLEDGMENT}

The authors wish to thank the ICIS Distinctive Signature at Idaho National Laboratory (INL) for the support of this work.

\section{REFERENCES}

[1] V. Venkatasubramanian, S. Katare, P. Patkar, and F.-P. Mu, "Spontaneous emergence of complex optimal networks through evolutionary adaptation," Computers and Chemical Engineering, vol. 28, pp. 1789-1798, 2004.

[2] V. Venkatasubramanian, "A theory of design of complex teleological systems: Unifying the darwinian and boltzmannian perspectives," Complexity, vol. 12(3), pp. 14-21, 2007.

[3] C. Rieger, D. Gertman, and M. McQueen, "Resilient control systems: next generation design research," in Proceedings of the IEEE Conference on Human System Interactions, 2009.

[4] T. Malik, R. Prasad, S. Patil, A. Chaudhary, and V. Venkatasubramanian, "Providing Scalable Data Services in Ubiquitous Networks," Lecture Notes in Computer Science, vol. 6193, pp. 445-457, 2010.

[5] A. Tero, S. Takagi, T. Saigusa, K. Ito, D. P. Bebber, M. D. Fricker, K. Yumiki, R. Kobayashi, and T. Nakagaki, "Rules for biologically inspired adaptive network design," Science, vol. 327, pp. 439-442, 2010.

[6] V. Venkatasubramanian, T. Malik, A. Giridhar, K. Villez, R. Prasad, A. Shukla, C. Rieger, K. Daum, and M. McQueen, "RNEDE: Resilient Network Design Environment," in Proceedings of the 3rd International Symposium on Resilient Control Systems (ISRCS), pp. 72-75, 2010.

[7] A.-L. Barabasi, and R. Reka Albert, "Emergence of scaling in random networks," Science, vol. 286, pp. 509-512, 1999.

[8] J. Travers, and S. Milgram, "An experimental study of the small world problem," Sociometry, vol. 32, pp. 425-443, 1969.

[9] S. Patil, S. Srinivasa, and V. Venkatasubramanian, "Classes of Optimal Network Topologies under Multiple Efficiency and Robustness Constraints," in Proceedings of the 2009 IEEE International Conference on Systems, Man, and Cybernetics, San Antonio, TX, USA, October 2009.

[10] S. Patil, S. Srinivasa, S. Mukherjee, A. R. Rachakonda, and V. Venkatasubramanian, "Breeding Diameter-Optimal Topologies for Distributed Indexes," Complex Systems, vol. 18, pp. 175-194, 2009.

[11] C. E. Thomas, "Fuel cell and battery electric vehicles compared," International Journal of Hydrogen Energy, vol. 34, pp. 6005-6020, 2009.

[12] M. A. Kromer, "Electric powertrains: Opportunities and challenges in the US light-duty vehicle fleet," PhD thesis. Massachusetts Institute of Technology, 2007.

[13] K. Galbraith, "Better Place unveils battery swap station," http://green.blogs.nytimes.com/2009/05/13/better-place-unveils-batteryswap-station/, 2009

[14] O. Berman, R. C. Larson, and N. Fouska, "Optimal location of discretionary service facilities," Transportation Science, vol. 26, 201211, 1992.

[15] A. Shukla, J. Pekny, and V. Venkatasubramanian, "An optimization framework for cost effective design of refueling station infrastructure for alternative fuel vehicles," Computers \& Chemical Engineering, In Press, Available online, 2011.

[16] G. Marfia, G. Pau, E. De Sena, E. Giordano, and M. Gerla, "Evaluating vehicle network strategies for downtown Portland: Opportunistic infrastructure and the importance of realistic mobility models," in Proceedings of the 1st international MobiSys workshop on mobile opportunistic networking, San Juan, Puerto Rico, pp. 47-51, 2007.

[17] S. Lawe, J. Lobb, A. W. Sadek, S. Huang and C. Xie, "TRANSIMS implementation in Chittenden County, Vermont. Development, Calibration, and Preliminary Sensitivity Analysis," Transportation Research Record: Journal of the Transportation Research Board, vol. 2132, pp. 113-121, 2009. 